\title{
Halide Perovskites - structural systematisation and what we learn from it
}

\author{
J. Breternitz ${ }^{1,2}$, S. Schorr ${ }^{1,3}$ \\ ${ }^{1}$ Helmholtz-Zentrum Berlin für Materialien uund Energie, Hahn-Meitner-Platz 1, 14109 Berlin, Germany, ${ }^{2}$ Universität Potsdam, \\ Karl-Liebknecht-Straße 24/25, 14476 Potsdam, Germany, 3Freie Universität Berlin, Malteserstraße 74-100, 12249 Berlin, Germany \\ joachim.breternitz@helmholtz-berlin.de
}

Hybrid halide perovskites have made a quite spectacular appearance in the field of photovoltaics, not only because device efficiency has shot over $25 \%$ within 10 years of their development [1], but also because of their specific behaviour that is yet to be fully understood. In structural terms, these materials compare to the oxide perovskites $A B \mathrm{O}_{3}$ in many respects [2] but also hold some features that are rather distinct and largely related to the molecular cation occupying the $A$-cation site. [3]

Therefore, a structural categorisation of these materials is greatly beneficial to understand the underlying principles of structure and property relationships. At the core of this work, we will present a fairly comprehensive group-subgroup relationship applied to halide perovskites and double perovskites deriving from the cubic perovskite aristotype in the form of a Bärnighausen tree [3] This is seconded with a discussion of the different distortion modes applying to halide perovskites: atom shifts, octahedral tilting and $A$-cation orientation, with the latter being a distinct mechanism in hybrid halide perovskites. Furthermore, we will elucidate the implications for the properties and phase transitions given the specific space group settings of the different crystal structures.

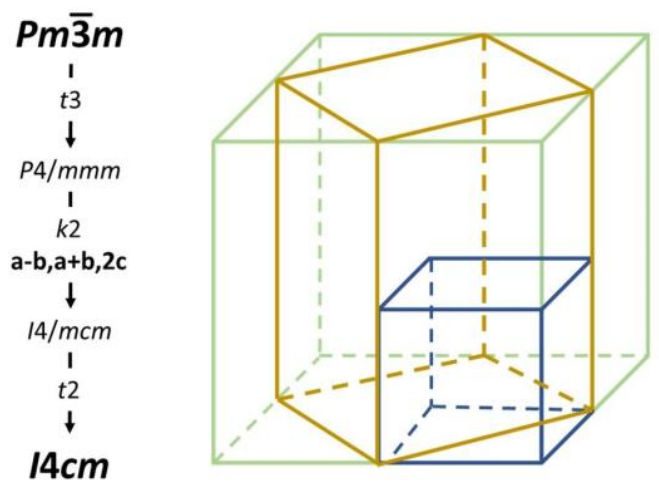

Figure 1. Group-subgroup relationship between the perovskite aristotype and the tetragonal room temperature space group of $\mathrm{MAPbI}_{3}$ along with the schematic change of unit cell edges between $P m \overline{3} m$ (blue), $I 4 \mathrm{~cm}$ (gold) and its non-standard setting $F 4 m c$ (green). [4]

We will highlight why the consideration of group-subgroup relationships in halide perovskites materials is not only of structuralsystematic interest, but allows direct assumptions on the device performance of perovskite solar cells. For this, we will uncover some of the physical implications of the structural relationships as they arise from the group-subgroup relationships - for instance twinning in the tetragonal phase of $\mathrm{MAPbI}_{3}$ [4] and a potential crystallographic explanation for the possibility of ferroelectricity in $\mathrm{MAPbI}_{3}[5]$.

[1] https://www.nrel.gov/pv/cell-efficiency.html, accessed 14/04/2021.

[2] Breternitz, J. \& Schorr, S. (2018). Adv. Energy Mater. 8, 1802366.

[3] Breternitz, J. (2021). Crystallography in Materials Science, edited by S. Schorr, C. Weidenthaler, Berlin: de Gruyter, in press.

[4] Breternitz, J., Tovar, M. \& Schorr, S. (2020). Sci. Rep. 10, 16613.

[5] Breternitz, J., Lehmann, F., Barnett, S. A., Nowell, H. \& Schorr, S. (2020). Angew. Chem. Int. Ed. 59, 424.

Keywords: halide perovskites; semiconductors; group-subgroup relations; twinning 